\title{
Cellulose fibers enable near zero-cost electrical sensing of water-soluble gases
}

Giandrin Barandun ${ }^{[a]}$, Matteo Soprani ${ }^{[a, b}$, c], Sina Naficy ${ }^{[a, d]}$, Max Grell ${ }^{[a]}$, Michael Kasimatis ${ }^{[a]}$, Kwan Lun Chiu $^{[a]}$, Andrea Ponzoni ${ }^{[b, c]}$ and Firat Güder*[a]

[a] Department of Bioengineering Imperial College London SW7 2AZ, London, UK

E-mail: guder@imperial.ac.uk

[b] Department of Information Engineering University of Brescia 25121, Brescia, Italy

[c] National Institute of Optics National Research Council 25121, Brescia, Italy

[d] School of Chemical and Biomolecular Engineering The University of Sydney NSW2006, Sydney, Australia 


\section{Table of Contents}

- SI-P1. Further discussions on the accuracy of the model proposed in the main text

$\circ \quad$ SI-P1.1. The case of $\mathrm{NH}_{3}$ and trimethylamine

- SI-P1.2. A model for predicting ionic conductivity

- SI-P2. Data analysis of food spoilage experiments

- SI-P3. Comparison with state-of-the-art electronic nose

\section{Results and Discussion}

\section{SI-P1. Further discussions on the accuracy of the model proposed in the main text}

\section{$\underline{\text { SI-P1.1. The case of } \mathrm{NH}_{3} \text { and trimethylamine }}$}

To further discuss the accuracy of our model (a detailed description of our model is shown below in part SI-P1.2), we would like to analyze a specific example involving $\mathrm{NH}_{3}$ and trimethylamine (TMA). According to our theoretical model, 10 parts-per-million (ppm) of ammonia should produce a similar change in conductivity to $20 \mathrm{ppm}$ of TMA, with a ratio of $\frac{\Delta \sigma_{N H_{3}}}{\Delta \sigma_{T M A}}=1.16\left(\Delta \sigma_{N H_{3}}\right.$ : change in conductivity produced by 10 ppm of $\mathrm{NH}_{3}, \Delta \sigma_{T M A}$ : the change in conductivity produced by 20 ppm TMA). Our experimental results for PEGS in Fig. 2A indicate that the ratio of change in conductivity for $\mathrm{NH}_{3}$ and TMA is approx. $40\left(\frac{\Delta G_{N H_{3}}}{\Delta G_{T M A}}=40\right)$. Hence, there is an order of magnitude mismatch between the calculated and experimental results for the concentrations mentioned above.

To understand whether the deviation from the calculations were originating from the use of paper (and not just a body of bulk water), we have performed the following experiment: We printed two carbon electrodes on a polymer substrate (marking transparency), submerged into $3 \mathrm{~mL}$ of deionized water and measured the change in conductivity in the presence of $10 \mathrm{ppm}$ of $\mathrm{NH}_{3}$ and $20 \mathrm{ppm}$ of TMA (Fig. S11) and took their ratio $\left(\frac{\Delta G_{N H_{3}}}{\Delta G_{T M A}}\right.$ ). In contrast to the experimental results produced by PEGS, the experiments involving bulk water produced results similar to the theoretical estimations with $\frac{\Delta G_{N H_{3}}}{\Delta G_{T M A}}=1.25$.

These results suggest that water adsorbed in paper and bulk water behave slightly differently for the same gases. This is probably because of the following reasons: 
- There may be interactions between the gases and cellulose matrix. Such evidence is found in cellulosic fiber treatment called 'mercerization' where physical and chemical properties of the cellulose fibers are modified using an alkaline solution, including ammonia. ${ }^{1}$ These modifications may influence the ionic conductivity.

- The surface bound layer of water has slightly different chemical properties than free (bulk) water.

- Structural changes of cellulose fibers have been reported when moisture content surpasses $3.5 \mathrm{wt} \%$. The density of paper does not behave monotonically with increasing levels of humidity. This suggests variable spatial distribution, size and connectivity of water reservoirs within the network of cellulose fibers, hence solution models may not likely reflect the paper sensor. ${ }^{2}$ 
$\underline{\text { SI-P1.2. A model for predicting ionic conductivity }}$

\section{Conductivity:}

$$
\sigma=n \times Z e \times u
$$

$n: \quad$ carrier concentration

Ze: $\quad$ charge number * elementary charge

$u: \quad$ carrier mobility

Additional conductivity due to dissociated ammonia:

$$
\sigma_{\mathrm{NH}_{3}}=e \times\left[n_{\mathrm{NH}_{4}^{+}} \times u_{\mathrm{NH}_{4}^{+}}+n_{\mathrm{OH}^{-}} \times u_{\mathrm{OH}^{-}}\right]
$$

Henry's solubility constant: $k_{H}^{\theta}$ :

$$
k_{H}^{\theta}=\frac{\left[N H_{3(a q)}\right]}{p_{N H_{3}}}
$$

$p_{\mathrm{NH}_{3}}$ : partial pressure of ammonia

Partial pressure $p_{\mathrm{NH}_{3}}$ :

$$
\frac{p_{\mathrm{NH}_{3}}}{p_{T o t}}=\frac{V_{N_{3}}}{V_{T o t}} \quad \Rightarrow \quad p_{N_{3}}=p_{T o t} \times \frac{V_{N_{3}}}{V_{T o t}}
$$

Dissociation constant $K_{B}$ :

$$
K_{B}=\frac{\left[\mathrm{NH}_{4}^{+}\right]\left[\mathrm{OH}^{-}\right]}{\left[\mathrm{NH}_{3(a q)}\right]}
$$

Assumption:

$$
\begin{aligned}
& {\left[\mathrm{NH}_{4}^{+}\right]=\left[\mathrm{OH}^{-}\right] \text {Solve equation (3) and (5) for }\left[\mathrm{NH}_{3(\mathrm{aq})}\right]:} \\
& {\left[\mathrm{NH}_{3(\mathrm{aq})}\right]=\frac{\left[\mathrm{NH}_{4}^{+}\right]^{2}}{K_{B}}}
\end{aligned}
$$

and

$$
\left[\mathrm{NH}_{3(a q)}\right]=H^{c p} \times p_{N H_{3}}
$$

Use equation (6) and (7) to get the additional carrier concentration $\left[\mathrm{NH}_{4}^{+}\right]$: 


$$
\left[\mathrm{NH}_{4}^{+}\right]=\sqrt{\mathrm{K}_{\mathrm{B}} \times \mathrm{H}^{c p} \times p_{\mathrm{NH}_{3}}}=\left[\mathrm{OH}^{-}\right]:=n_{\mathrm{NH}_{4}^{+}}=n_{\mathrm{OH}^{-}}
$$

Insert $\left[\mathrm{NH}_{4}^{+}\right]$into equation (2) to get the additional conductivity $\sigma_{\mathrm{NH}_{3}}$ :

$$
\sigma_{N_{3}}=e \times \sqrt{K_{B} \times H^{c p} \times p_{N H_{3}}} \times\left[u_{N_{4}^{+}}+u_{O H^{-}}\right]
$$

\section{Constants $^{3,4}$}

\section{Dissociation constant:}

Ammonia

$$
K_{B}=10^{-4.76} \frac{\mathrm{mol}}{\mathrm{l}}
$$

Trimethylamine

$$
K_{B}=10^{-4.2} \frac{\mathrm{mol}}{\mathrm{l}}
$$

$\underline{\text { Henry's solubility constant: }}$

Ammonia

$$
H^{c p}=67.75 \frac{\mathrm{mol}}{l \times b a r}
$$

Trimethylamine

$$
H^{c p}=9.50 \frac{\mathrm{mol}}{l \times b a r}
$$

Mobility:

$$
\begin{array}{ll}
\text { Ammonium } & u_{\mathrm{NH}_{4}^{+}}=7.62 \times 10^{-8} \frac{\mathrm{m}^{2}}{\mathrm{~s} \times \mathrm{V}} \\
\text { Hydroxide } & u_{\mathrm{OH}^{-}}=20.64 \times 10^{-8} \frac{\mathrm{m}^{2}}{\mathrm{~s} \times V} \\
\text { Trimethylammonium } & u_{\mathrm{NC}_{3} \mathrm{H}_{10}^{+}}=3.56 \times 10^{-8} \frac{\mathrm{m}^{2}}{\mathrm{~s} \times V}
\end{array}
$$

\section{SI-P2. Data analysis of food spoilage experiments}

We obtained our raw data from an Arduino DUE ADC port (10 bit) and scaled it to consider the different gain resistors we used in our transimpedance amplifying setup $\stackrel{1}{1}$. This gave us the data as shown in Fig. S6 A+B.

We applied a moving average filter before we looked for local maxima or minima in our data. The red circles in Fig. S6 A+B indicate the position where the $1^{\text {st }}$ derivative is zero. We chose these points as our conductance

\footnotetext{
${ }^{1}$ Gain scale vector: $[1 ; 3.0551 ; 15.0739 ; 494.4227]$
} 
reference value $\left(G_{0}\right)$ to normalize each sensor individually. These turning points show where the sensor started reaching equilibrium with the container's atmosphere. This means, external factors (e.g. added water, temperature) do not influence the sensors anymore. We normalized the data by subtracting and dividing by the reference value $\left(\left[\mathrm{G}-\mathrm{G}_{0}\right] / \mathrm{G}_{0}=\Delta \mathrm{G} / \mathrm{G}_{0}\right)$. This gave data that indicated the change of the conductance over time in relation to a reference value (Fig. S6 C+D). We then normalized the response to $100 \mathrm{~g}$ per sample. In this case the raw data was for 40g cod fish (Fig. S6 A) and 20g chicken breast (Fig. S6 B) respectively. Averaging over the four sensors in the containers with food samples and the two sensors in the water (control) boxes, gave us the data presented in Fig. 5 in the main text.

\section{SI-P3. Comparison with state-of-the-art electronic nose}

To better frame the effectiveness of the proposed paper sensors within state-of-the-art technologies, we tested metal oxide (MOX) gas sensors in parallel with paper sensors to track the degradation of cod over time.

We chose MOX sensors because of their well-established sensing capability, which has been widely demonstrated in a variety of applications.,

The experimental setup was the same as used for the food experiment with the paper gas sensor. We kept the MOX device inside a container $(180 \mathrm{~mL})$ together with two paper-based sensors. Both technologies were tested under the same conditions and against the same target.

Since MOX are non-specific sensors, i.e. they respond to a broad range of chemicals, the electrical resistance of a single MOX device is not suitable to track the target in complex atmospheres, such as those developed by decomposing food. These MOX sensors were, therefore, exploited in a sensor array configuration (so called "electronic nose"). We applied temperature modulation protocols to achieve the desired selectivity and the sensor response is depicted as a Principal Component (PC) plot in Fig. S7.7 The sensor's response was acquired every 20 seconds and each data point corresponds to a single measurement. For simplicity, a set of 20 data points acquired every 6 hours in a frame of 400 seconds is plotted. This data formed six different clusters. From the clusters' arrangement it is possible to observe a trend that is representative to the cod degradation and the 
capability of MOX sensors to track it. It can be observed that clusters are well separated for the first 18 hours.

The distance between consecutive clusters diminished over time until the clusters were almost overlapping after 18 hours. This indicates the capability of the MOX device to distinguish among the different degradation degrees during the initial stages and shows that the performance of the MOX sensors suffers as the spoilage gas concentration increases. This may be reasonably ascribed to saturation effects. ${ }^{8}$ This saturation occurs when the total microbial concentration value is around $10^{8}-10^{9} \mathrm{CFU} / \mathrm{g}$ (colony forming units per gram), according to the microbial control experiment.

Methods for metal oxide sensors: We used a commercial platform (Minimox from JLM Innovation GmbH) equipped with two micromachined MOX sensors: TGS8100 (Figaro) and CSS801 (CCMOSS). We applied a square wave to the sensor heaters, to give a warm period of 10 seconds at voltage $V_{\text {heater }}=2.31 \mathrm{~V}$ and a cold period of 10 seconds with $V_{\text {heater }}=1.65 \mathrm{~V}$. We measured the sensor resistance with a sampling rate of 40 samples $/ \mathrm{s}$. Since the resistance value of a single MOX gas sensor is unsuitable to track the complex processes underlying food spoilage, the response of MOX sensors is retrieved by periodically warming and cooling the sensors through the embedded heater. This activates and freezes the interaction between gaseous molecules and the metal oxide surface, producing a resistance vs. time curve. ${ }^{9}$

In this work, the two metal oxide gas sensors were excited according to the same protocol: We applied a square wave of period 20 seconds, duty cycle $50 \%$ and voltage values of $2.31 \mathrm{~V}$ and $1.65 \mathrm{~V}$. Fig. S14 shows the resistance over one wave cycle of the CSS801 sensor measured during cod spoilage experiments at the beginning of the experiment (Fig. S14 A) and after 24 hours (Fig. S14 B). The resistance increased during the cold period and decreased during the warm period. The curves differ in terms of amplitude and shape, which reflects the different composition of the gas phase developed by fish over time. To characterize the shape of these curves, we used the following parameters:

- $\Delta \mathbf{R}_{\text {cold-hot }}$ is the resistance variation between the cold and the hot period. More precisely, it is calculated as the difference between the sensor resistance measured at the end of the cold period and the resistance measured at the start of the warm period after 0.2 seconds. 
- $\Delta \mathbf{R}_{\text {cold }}$ is the resistance variation within the cold period. It is calculated as the difference between the sensor resistance measured at the end of the cold period and the resistance measured at the beginning (after 0.2 seconds) of the same period.

We calculated these for each sensor and used a principal component analysis algorithm (PCA-function of MATLAB) on the data. 


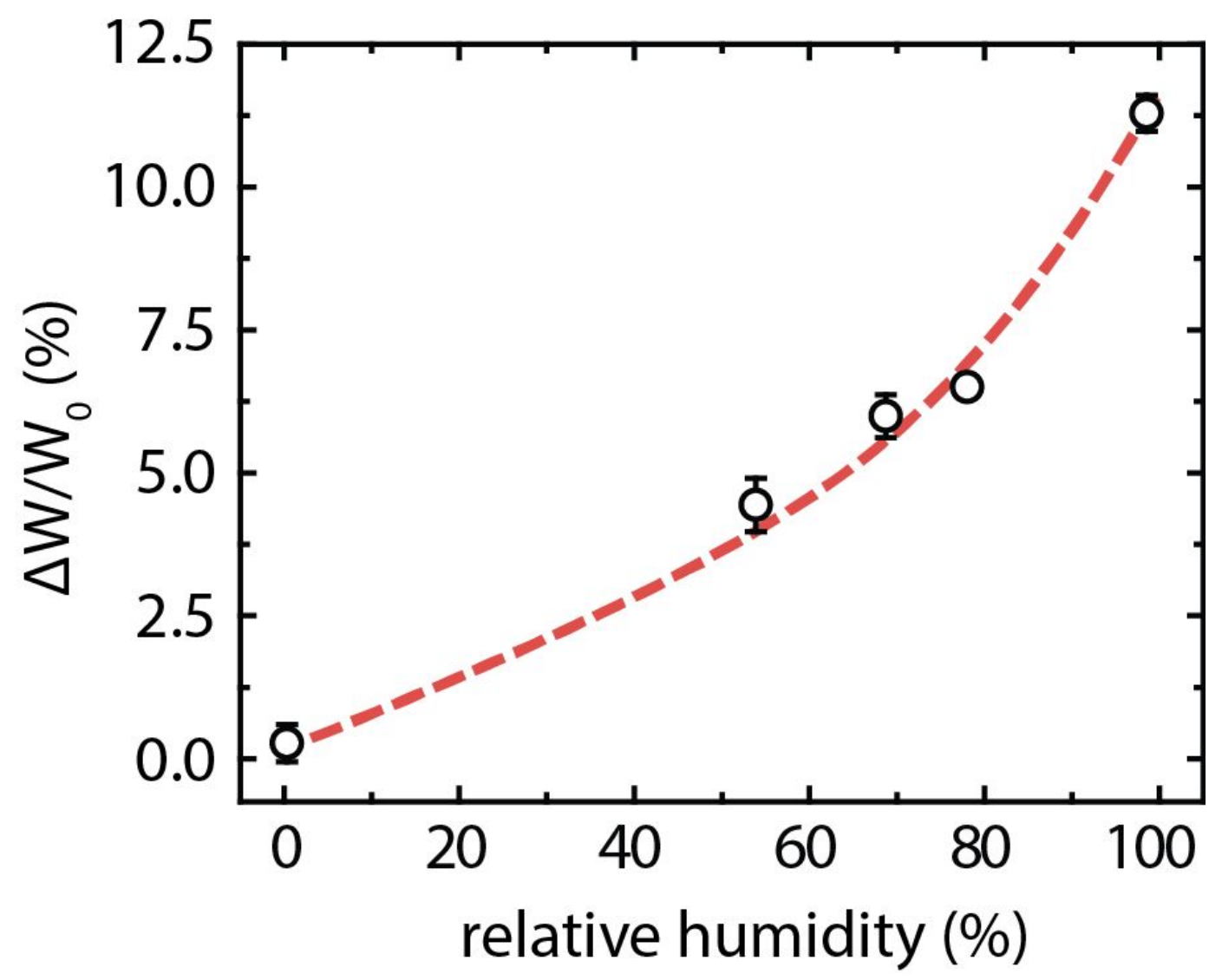

Figure S1. The weight percentage change $\left(\Delta \mathrm{W} / \mathrm{W}_{0}\right)$ of paper at different RH levels compared to the paper weight at $0 \% \mathrm{RH}(n=3)$. 


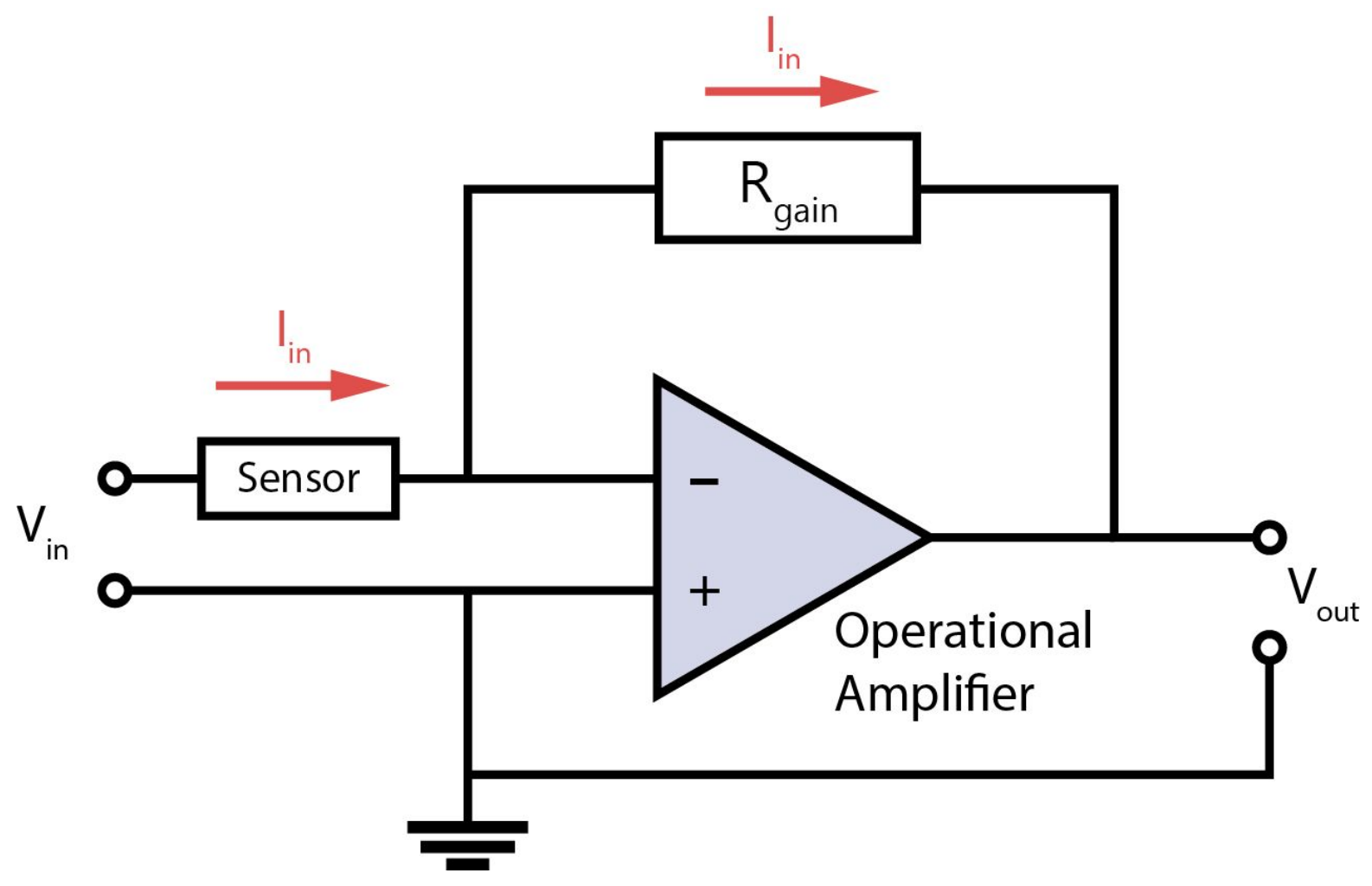

Figure S2. Operational amplifier-based transimpedance amplifier which converts the current passing through the sensor to a voltage. The sinusoidal signal is applied at $V_{\text {in }}$ and current through the sensor $\left(I_{\text {in }}\right)$ is converted to a voltage $\left(V_{\text {out }}\right)$ with a certain ratio, depending on the gain resistor $\left(R_{\text {gain }}\right)$. The input current is amplified according to:

$$
V_{\text {out }}=-\boldsymbol{R}_{\text {gain }} \times \boldsymbol{I}_{\text {in }}
$$

The circuit schematics for the entire read-out device are shown in Fig. S12 and S13 in detail. 


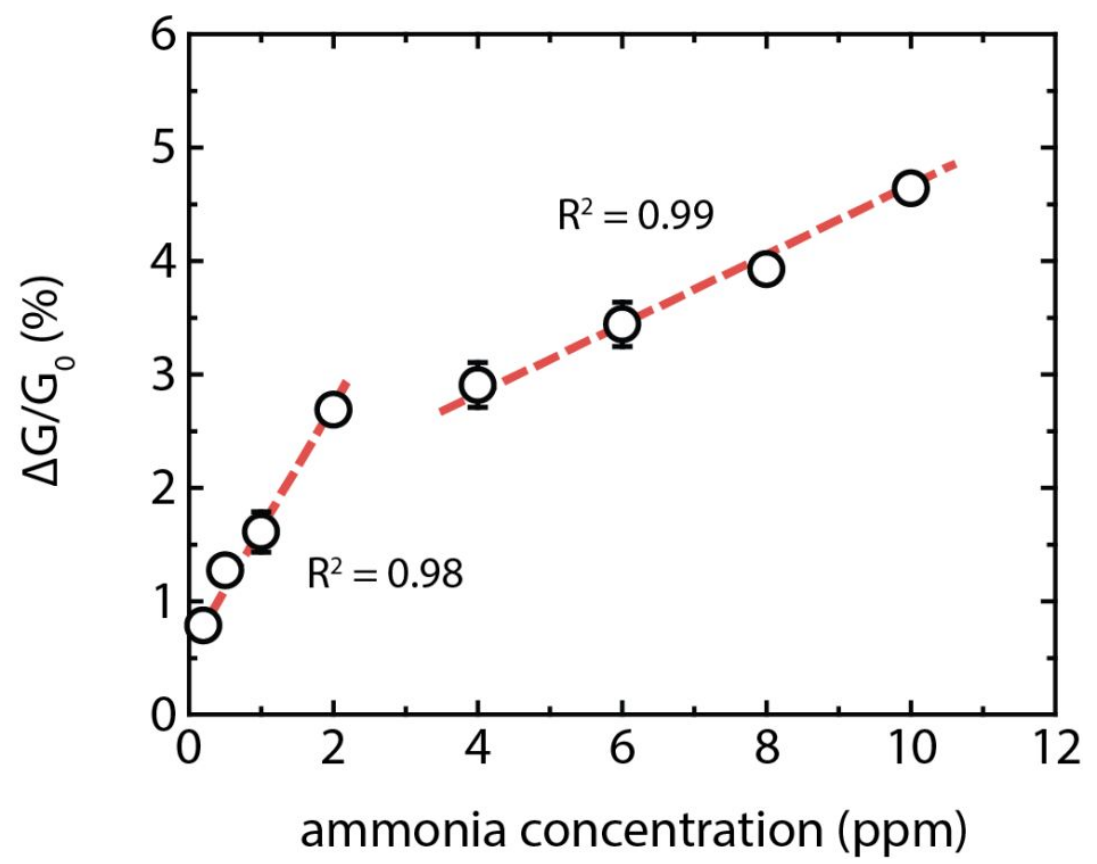

Figure S3. The average electrical response of the PEGS to ammonia gas concentrations from $0.2 \mathrm{ppm}$ to $10 \mathrm{ppm}$. Sensor response exhibited non-linearity at lower concentrations. 


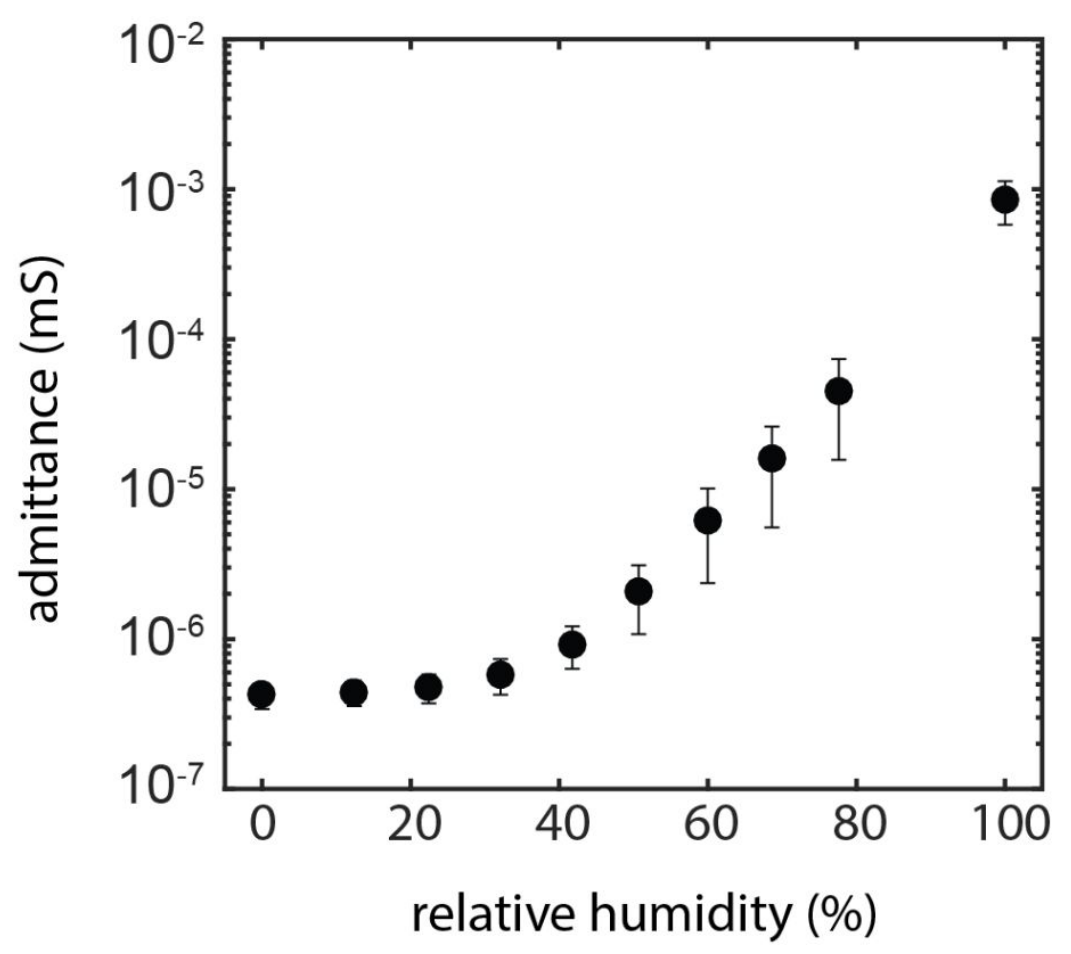

Figure S4. Admittance of the paper gas sensor over the RH range from 0-100 \% at a frequency of $20 \mathrm{~Hz}$. The error bars show the standard deviation $(n=3)$. 


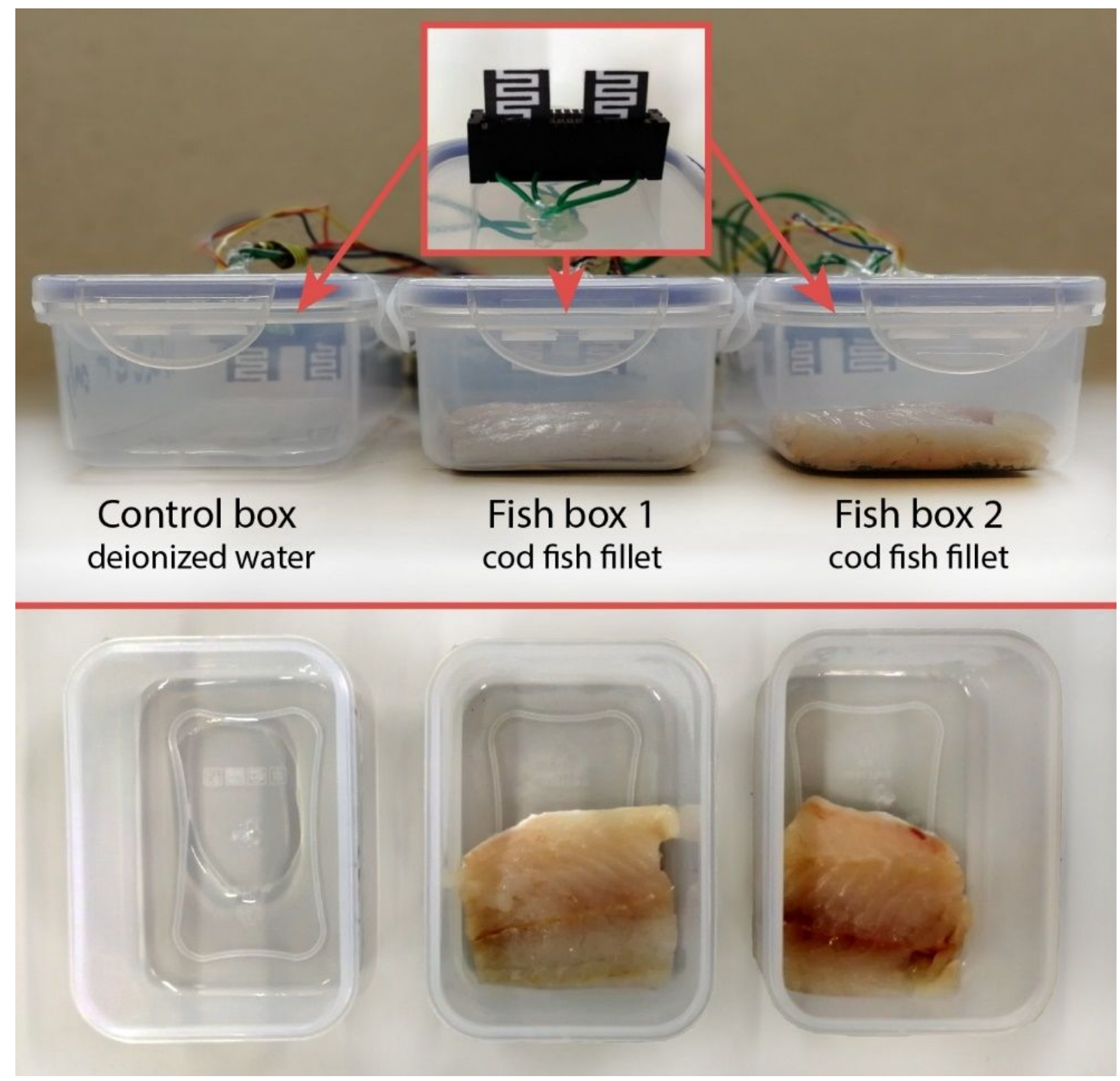

Figure S5. The three containers used for the fish and poultry monitoring experiments. One box contains water as a control and two boxes contain the meat (either cod fish fillet or chicken breast). Each container includes two paper sensors that are plugged into a card edge connector on the lid and a HIH-5030 type commercial humidity sensor. 

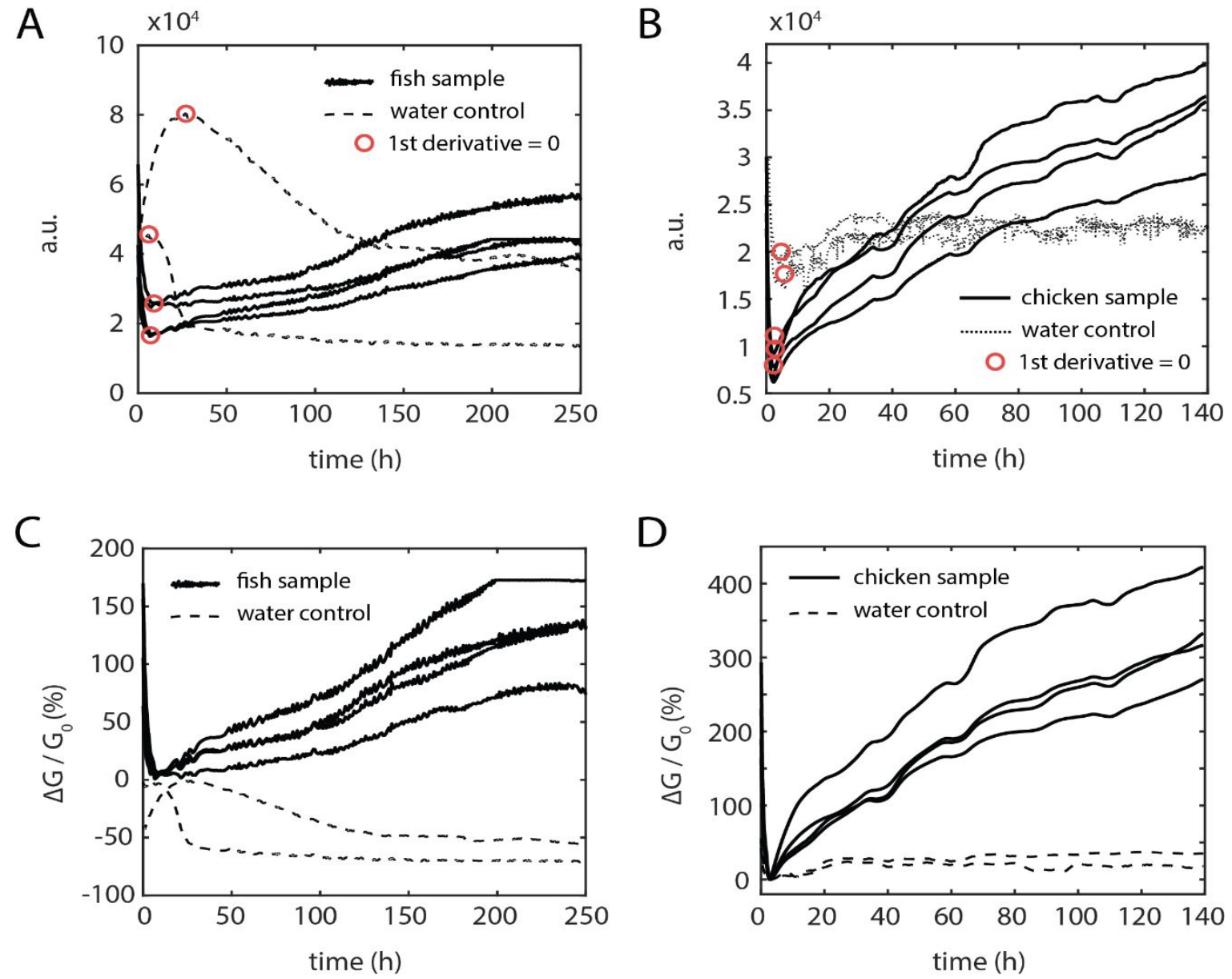

Figure S6. Raw data of the food monitoring experiments. (A) Signal of paper gas sensors in three different containers inside a fridge, two containing a cod fish sample $(40 \mathrm{~g})$ and one box containing water only. We chose the point where the $1^{\text {st }}$ derivative became zero to be the reference conductance value $\left(G_{0}\right)$ for each sensor (red circles). (B) The corresponding data for a chicken sample (20g) at room temperature. (C) We subtracted and divided the data from $(\mathrm{A})$ by its reference point $\left(1^{\text {st }}\right.$ derivative $\left.=0\right)$. This achieved normalized data indicating a change in conductance in percentage (\%) compared to the reference value. In a last step we normalized to a fish sample of 100g to achieve our final plot (Fig. 5). (D) Corresponding data for chicken. For the control sensor signal we additionally used a moving average filter $(n=200)$ before we normalized the data. 


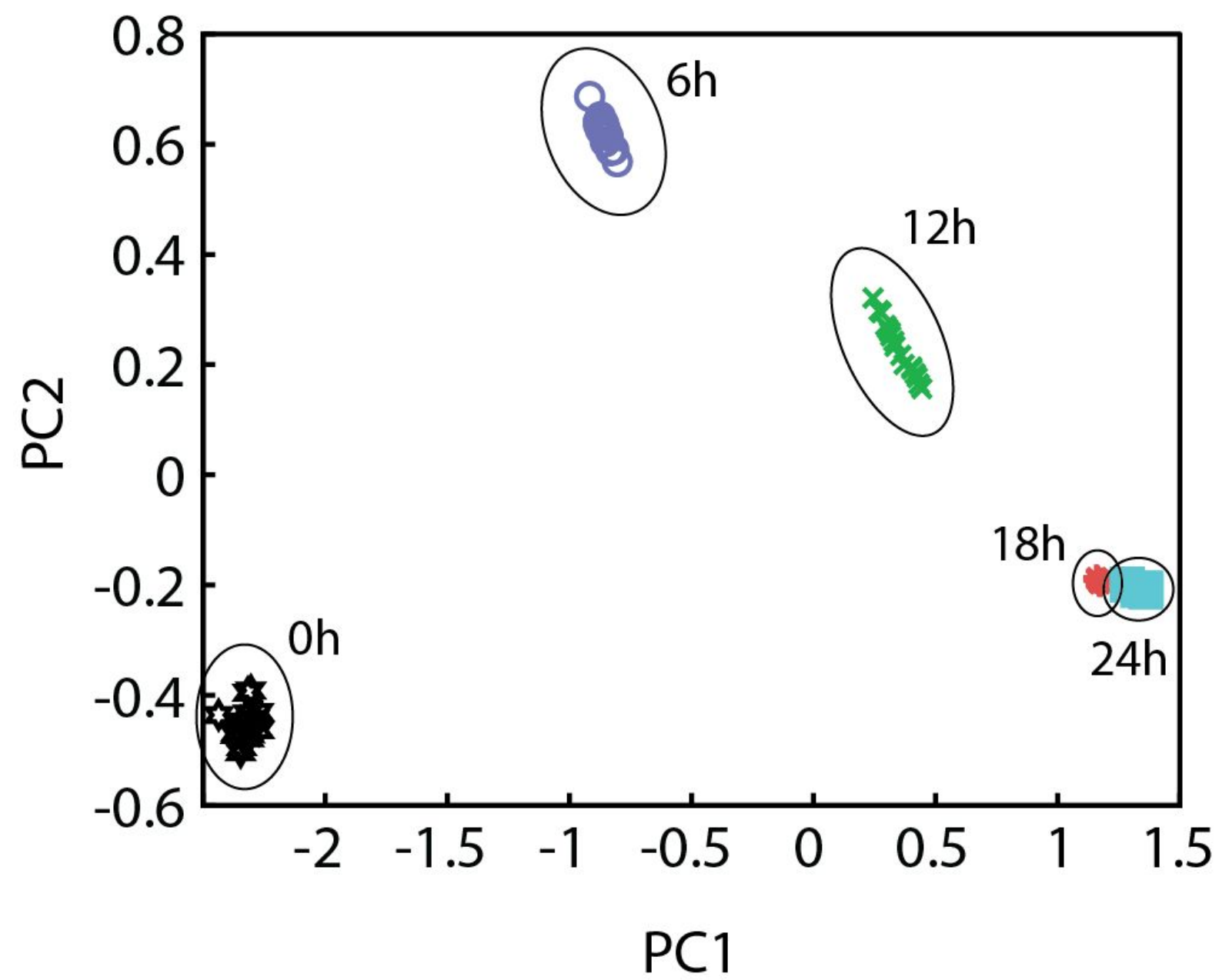

Figure S7. Principal component plot of the MOX sensor response to the volatile gases developed by a cod sample in the same setup as Fig. 5. The sensor response is plotted at five different times $(t=0 \mathrm{~h}$ to $\mathrm{t}=24 \mathrm{~h})$. The distribution of the data points is a function of volatile gases developing over time because of fish spoilage. Separation among the clusters shows the capability of the MOX technology to discriminate among different spoilage levels. Saturation effects (reduced cluster separation with increasing time) are visible after 18 hours. 


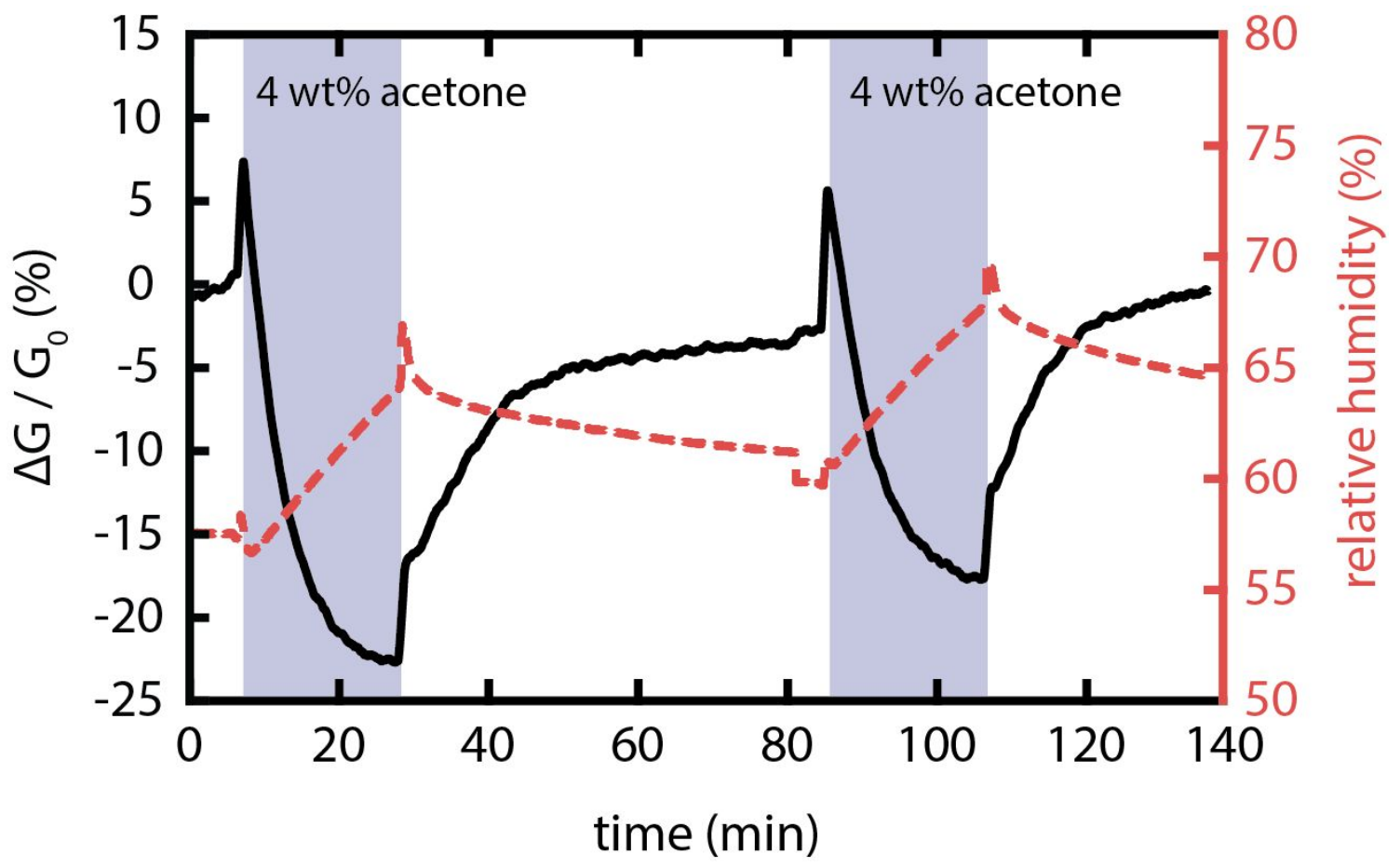

Figure S8. The response of PEGS (solid black) and commercial relative humidity sensor (dashed red) to an atmosphere of $4 \mathrm{wt} \%$ acetone in water. Exposure time (shaded band) and purge time were $20 \mathrm{~min}$ and $60 \mathrm{~min}$, respectively. The water-miscible acetone vapor produces a complex response in both PEGS and the commercial relative humidity sensor. Although we do not know the exact origin of this behavior, it is probably due to a change in surface tension of the adsorbed layer of water on both devices when the acetone vapor mixes with the liquid water on the surface. This phenomenon will be a topic of a future study. 


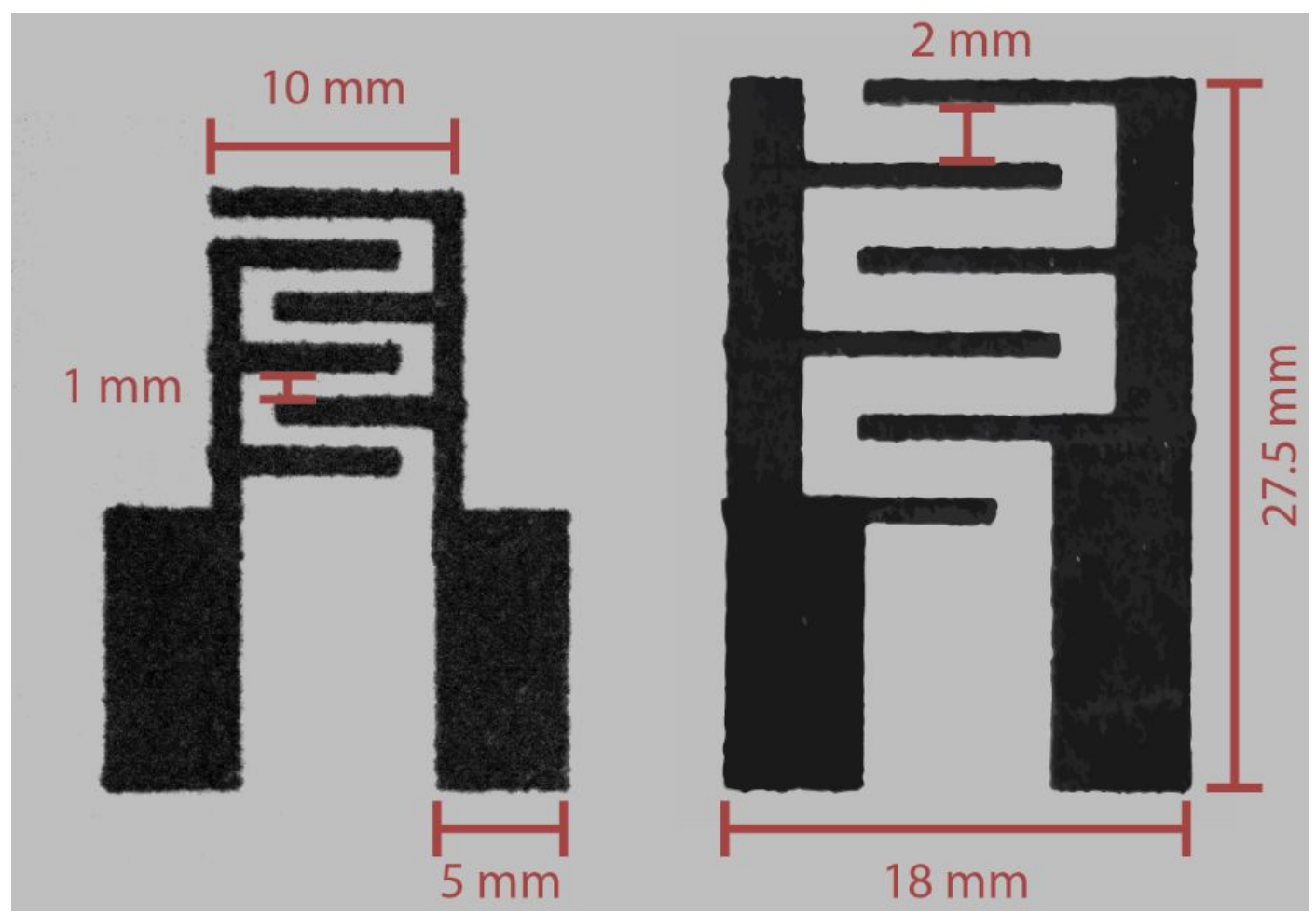

Figure S9. Optical photography of the carbon ink electrodes and their dimensions for sensor characterization (left) and food (right) experiments. 


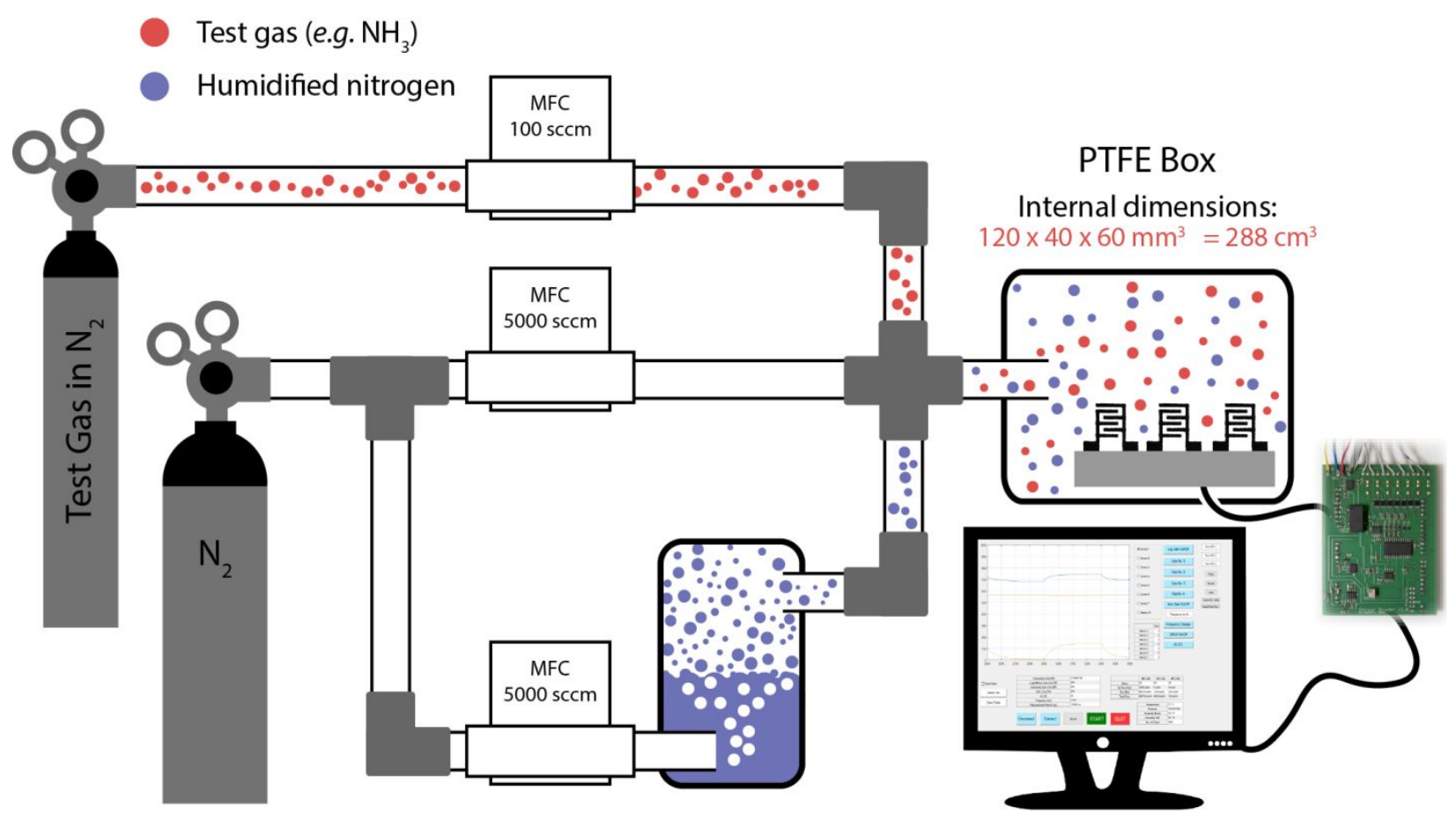

Figure S10. Setup for the sensor characterization experiment. One part of the nitrogen flow bubbles through water and gets humidified (blue circles). The test gas (red circles) is mixed with the dry and humidified nitrogen. The mixing ratio is highly controllable using mass flow controllers (MFCs) to achieve the desired RH and test gas concentration inside the PTFE box containing the sensors. The range of the different MFCs is indicated in standard cubic centimeters per minute ( $\mathrm{sccm}$ ). The sensor signal is amplified and processed on a homemade read-out device and plotted on a computer. 

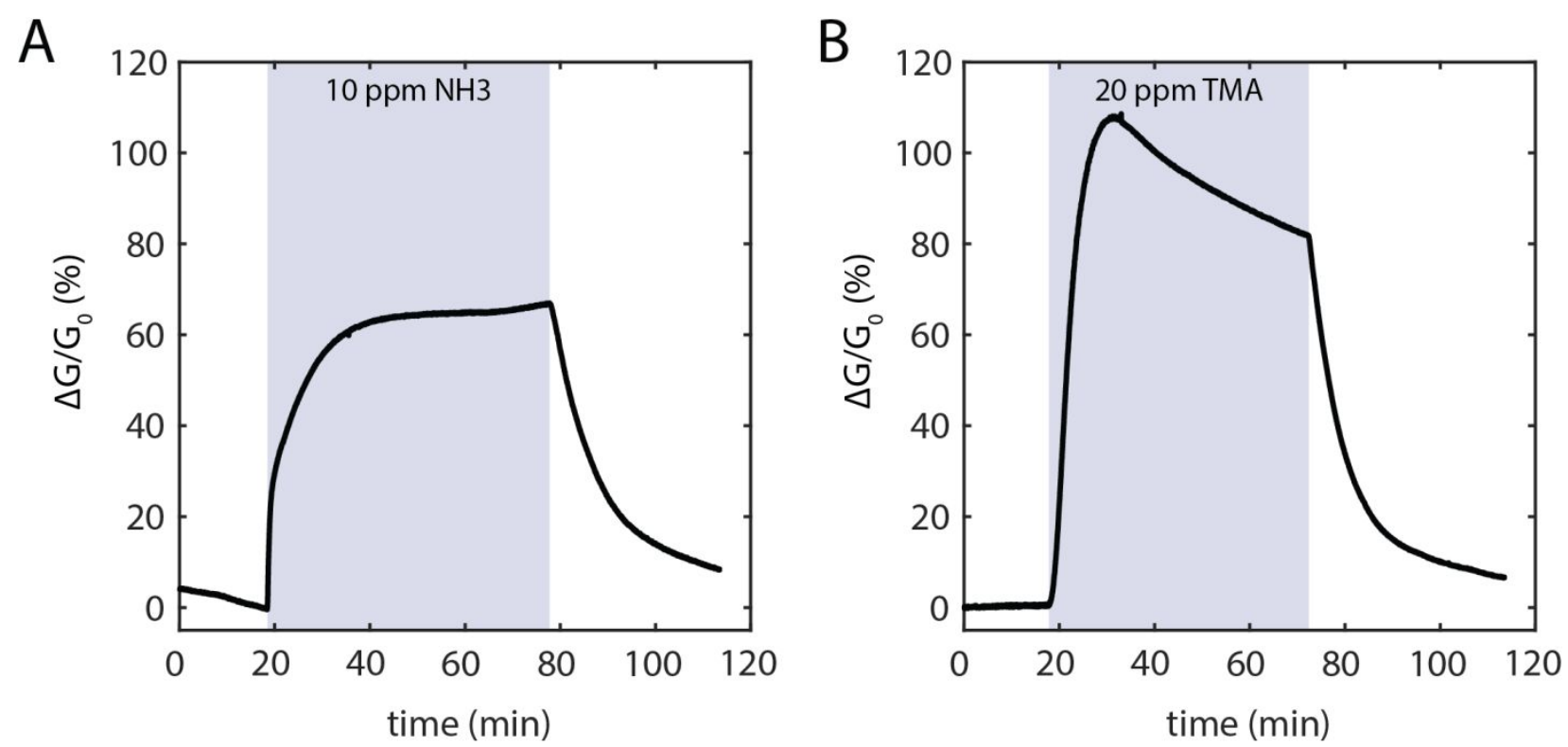

Figure S11. The signal response of two carbon electrodes in bulk deionized water:

(A) to $10 \mathrm{ppm}$ ammonia over ca. $60 \mathrm{~min}(n=2)$;

(B) to 20 ppm TMA over ca. $60 \min (n=2)$.

The blue bands show when the test gas $\left(\mathrm{NH}_{3}\right.$ or TMA) was present. 


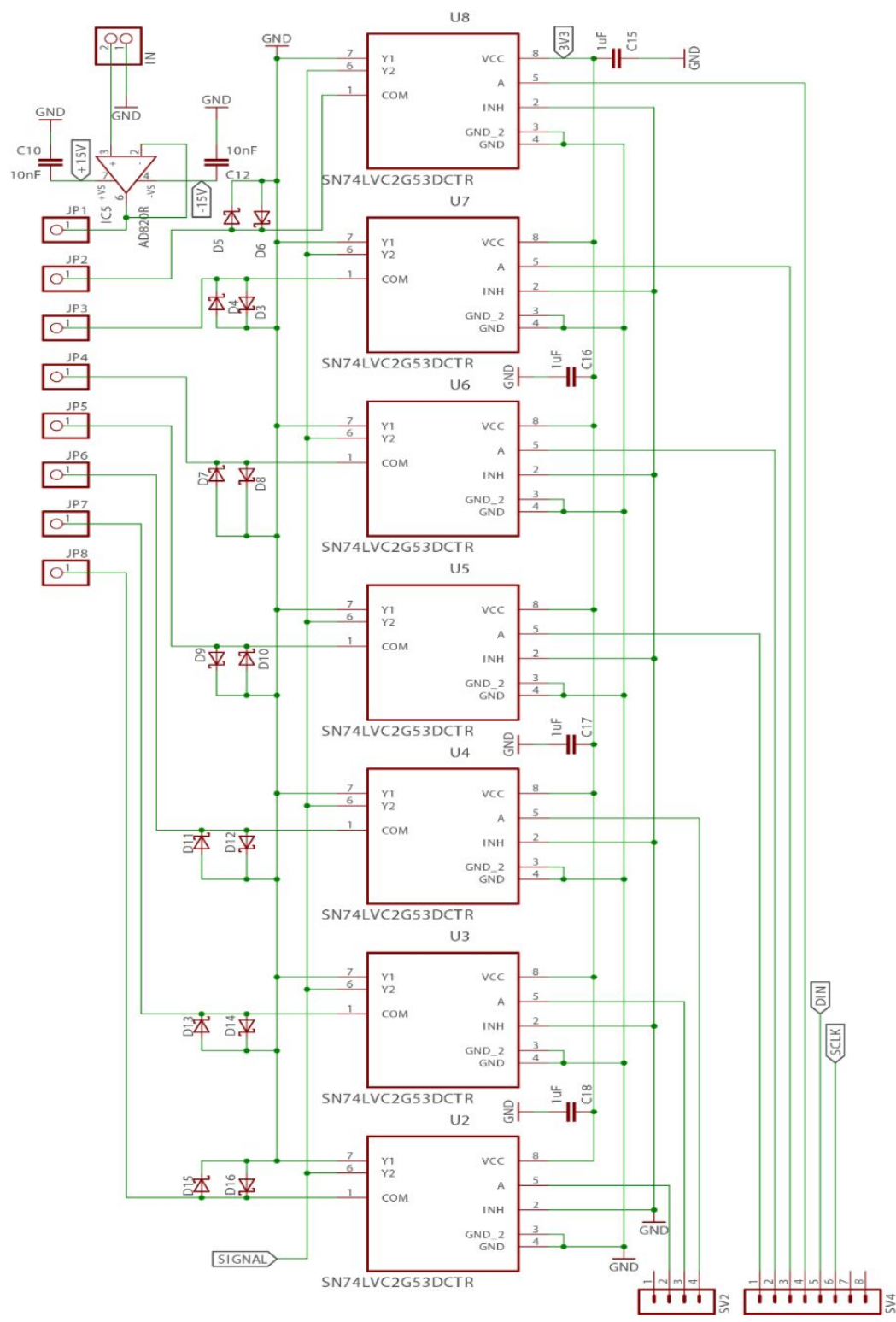

Figure S12. Read-out device schematics, part 1; A sinusoidal wave from a function generator (IN) goes through an operational amplifier (AD820) in a voltage follower configuration and is applied to seven sensors simultaneously (JP1). The sensors outputs (JP2-JP8) are controlled by ultra-fast switches (SN74LVC2G53DCTR) and one at a time is forwarded to the read-out setup (SIGNAL). 


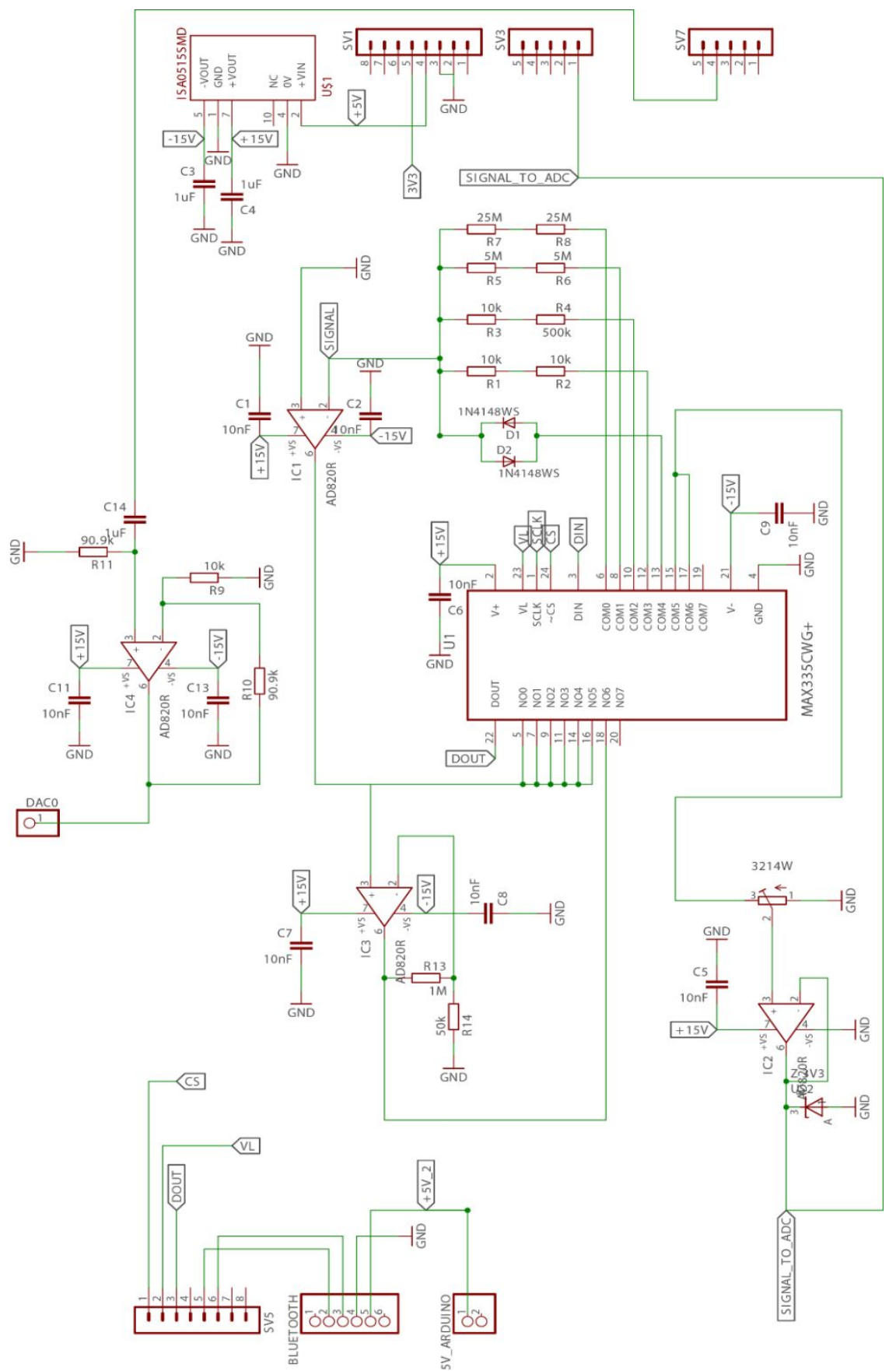

Figure S13. Read-out device schematics, part 2; SIGNAL follows through a transimpedance configuration (IC1) where the gain resistor is chosen by a multiplexer (MAX335CWG+) for the signal to fit the voltage range of the microcontroller. It is further adjusted using a voltage divider (3214W) and read by an Arduino Due (SIGNAL_TO_ADC) after a voltage follower (IC2). 

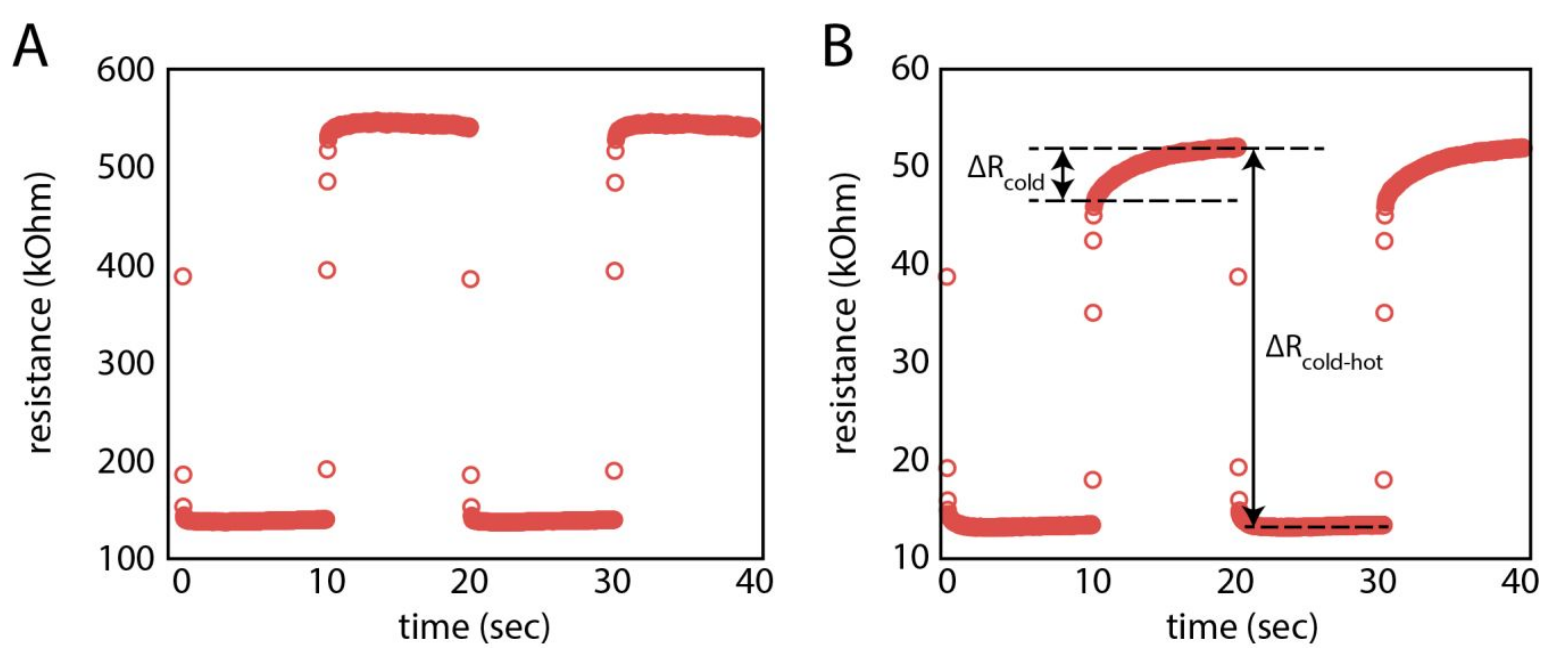

Figure S14. MOX sensor signal as electrical resistance over time. These signals are recorded during cod experiments. (A) Two cycles of a hot and cold period of the MOX sensor at the start of the experiment $(\mathrm{t}=0 \mathrm{~h})$. (B) Similarly, two cycles of a hot and cold period of the MOX sensor after $24 \mathrm{~h}$. The parameter $\Delta \mathrm{R}_{\text {cold }}$ is used to quantify the shape of the curve and $\Delta \mathrm{R}_{\text {cold-hot }}$ quantifies the difference in resistance between the cold and the hot period of the measurement cycle. 
Table S1. Henry's law solubility constant ${ }^{4}$ and dissociation constants ${ }^{3}$ for various gases

\begin{tabular}{|c|c|c|c|}
\hline Gas & $\begin{array}{c}\text { Solubility } \\
\left({\left.\text { mol } \times \mathrm{kg}^{-1} \text { bar }^{-1}\right)}\right.\end{array}$ & $\mathrm{p} K_{b}$ & $\mathrm{p} K_{a}$ \\
\hline Ammonia & 57 & 4.76 & \\
\hline Trimethylamine & 9.6 & 4.2 & \\
\hline Carbon Dioxide & 0.034 & & $\begin{array}{c}6.35 \\
10.33\end{array}$ \\
\hline Carbon Monoxide & 0.00097 & - & - \\
\hline Hydrogen Sulfide & 0.087 & & 7 \\
\hline
\end{tabular}


Movie S1. We bypassed an NFC tag with a resistor and a paper gas sensor (Fig. 7). The sensor had a DC

resistance of $1640 \mathrm{k} \Omega$ and a capacitance of $0.5 \mathrm{nF}$ with no ammonia gas present. We kept the modified tag in a $180 \mathrm{~mL}$ container and tapped a reader (smartphone HUAWEI P9) to detect it. The communication between tag and reader worked when no ammonia gas was present. We added $15 \mathrm{~mL}$ of $10 \%$ ammonia solution to the container and the DC resistance and capacitance of the sensor changed to $80 \mathrm{k} \Omega$ and $7.5 \mathrm{nF}$. The tag was not detected by the reader anymore. This was because the integrated circuit of the tag was bypassed with a PEGS and did not receive enough power to communicate with the reader. We demonstrate the paper-based sensor as an on/off detector for ammonia gas.

\section{References}

(1) Sakran, M. A. Effect of lonizing Radiation on the Spectral and Electrical Properties of Clean NH3-Mercerized Cotton Fabric Strips. J. Radioanal. Nucl. Chem. Lett. 1996, 213 (1), 51-63. https://doi.org/10.1007/BF02162485.

(2) Sapieha, S.; Inoue, M.; Lepoutre, P. Conductivity and Water Sorption in Paper. J. Appl. Polym. Sci. 1985, 30 (3), 1257-1266. https://doi.org/10.1002/app.1985.070300329.

(3) Lide, D. R. CRC Handbook of Chemistry and Physics, 89th Edition (Internet Version 2009); CRC Press: Boca Raton, 2009.

(4) Sander.R. Compilation of Henry's Law Constants for Inorganic and Organic Species of Potential Importance in Environmental Chemistry; 1999.

(5) Kim, S.-J.; Choi, S.-J.; Jang, J.-S.; Cho, H.-J.; Kim, I.-D. Innovative Nanosensor for Disease Diagnosis. Acc. Chem. Res. 2017, 50 (7), 1587-1596. https://doi.org/10.1021/acs.accounts.7b00047.

(6) Moon, H. G.; Shim, Y.-S.; Kim, D. H.; Jeong, H. Y.; Jeong, M.; Jung, J. Y.; Han, S. M.; Kim, J. K.; Kim, J.-S.; Park, H.-H.; et al. Self-Activated Ultrahigh Chemosensitivity of Oxide Thin Film Nanostructures for Transparent Sensors. Sci. Rep. 2012, 2, 588. https://doi.org/10.1038/srep00588.

(7) Hierlemann, A.; Gutierrez-Osuna, R. Higher-Order Chemical Sensing. Chemical Reviews. American Chemical Society 2008, pp 563-613. https://doi.org/10.1021/cr068116m.

(8) Berna, A.; Berna; Amalia. Metal Oxide Sensors for Electronic Noses and Their Application to Food Analysis. Sensors 2010, 10 (4), 3882-3910. https://doi.org/10.3390/s100403882.

(9) Lee, A. Temperature Modulation in Semiconductor Gas Sensing. Sensors Actuators B Chem. 1999, 60 (1), 3542. https://doi.org/10.1016/S0925-4005(99)00241-5. 